\title{
Leveraging Chaos for Wave-Based Analog Computation: Demonstration with Indoor Wireless Communication Signals
}

\author{
Philipp del Hougne ${ }^{1, *}$ and Geoffroy Lerosey ${ }^{2}$ \\ ${ }^{1}$ Institut Langevin, CNRS UMR 7587, ESPCI Paris, PSL Research University, \\ 1 rue Jussieu, 75005 Paris, France \\ ${ }^{2}$ Greenerwave, ESPCI Paris Incubator PC'up, 6 rue Jean Calvin, 75005 Paris, France
}

(Received 8 May 2018; revised manuscript received 13 August 2018; published 30 November 2018)

\begin{abstract}
In sight of fundamental thermal limits on further substantial performance improvements of modern digital computational processing units, wave-based analog computation is becoming an enticing alternative. A wave, as it propagates through a carefully tailored medium, performs the desired computational operation. Yet, the necessary designs are so intricate that experimental demonstrations will necessitate further technological advances. Here, we show that, counterintuitively, the carefully tailored medium can be replaced with a random medium, subject to an appropriate shaping of the incident wave front. Using tunable metasurface reflect-arrays, we demonstrate our concept experimentally in a chaotic microwave cavity. We conclude that off-the-shelf wireless communication infrastructure in combination with a simple reflect-array suffices to perform analog computation with Wi-Fi waves reverberating in a room.
\end{abstract}

DOI: 10.1103/PhysRevX.8.041037

Subject Areas: Complex Systems, Condensed Matter Physics, Optics

\section{INTRODUCTION}

The first steps in the history of computation were analog mechanical devices designed for specific tasks such as the Thomson brothers' harmonic analyzer to determine an input's Fourier representation [1]. With the dawn of the digital age, electronic general-purpose processors took over for good. For a long time, their Moore's law march of exponential performance improvements made any efforts to design specific-purpose devices redundant [2]. Nowadays, the underlying approach of squeezing more and more transistors into a unit chip area is confronted with fundamental thermal limits: Already today, only a certain number of transistors on a processor can be powered at any given time to avoid a silicon meltdown [3]. This so-called dark-silicon problem recently sparked renewed interest in specific-purpose units conceived to efficiently meet specific computation needs $[4,5]$. One route pursued by all major technology companies is to design specialized hardware devices based on application-specific integrated circuits (ASICs) or field-programmable gate arrays (FPGAs), capable of performing linear operations like matrix-vector multiplications very efficiently. Yet, at some point, the same

"philipp.delhougne@gmail.com

Published by the American Physical Society under the terms of the Creative Commons Attribution 4.0 International license. Further distribution of this work must maintain attribution to the author(s) and the published article's title, journal citation, and DOI. thermal constraints will thwart further performance improvements, too. There is hence a clear motivation to identify fundamentally different computation paradigms. One enticing idea is wave-based analog computation (WBAC), which proposes to swap the electronic circuits for materials with carefully tailored scattering properties such that the computational operation is performed on an incident wave front as it interacts with the material-see Fig. 1(a).

Some examples of such materials performing certain operations are well-known free-space optical components: A lens yields the Fourier transform of an impinging wave front [6,7], but the setup remains very bulky. Recent efforts on identifying appropriate materials for WBAC can be summarized in three groups that bring about different challenges with respect to (re)configurability and fabrication practicality:

(i) Leverage a specific physical effect to perform one specific operation enabled by that effect.A notable and experimentally demonstrated example of this approach uses interference phenomena arising from surface plasmon excitation to perform spatial differentiation [8]. While this specific operation is certainly of high relevance to highthroughput real-time image processing, it depends on the physics of the leveraged effect and, thus, inherently does not enable one to configure the device to perform a desired operation.

(ii) Design a metamaterial block to perform one specific desired operation.-A suitably designed metamaterial block was numerically shown to enable the 
(a)

Input wave front $X$
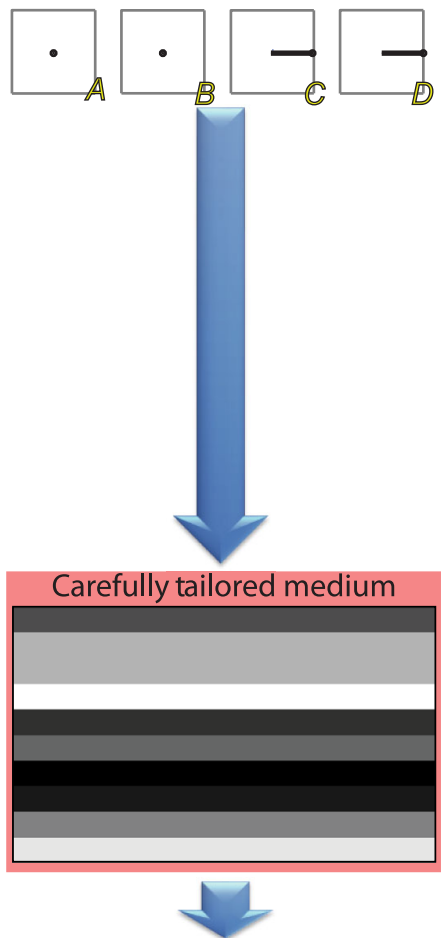

Output wave front $Y$ (b)

Input wave front $X$

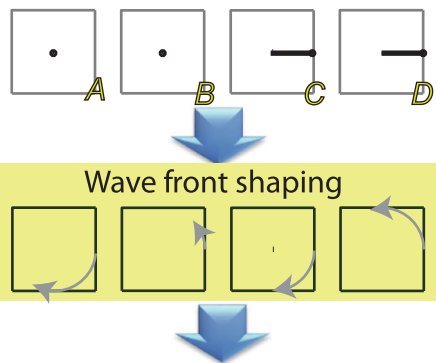

Shaped input wave front
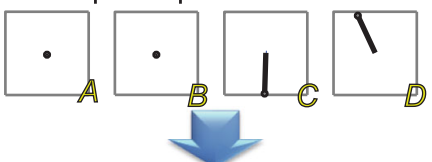

Random medium

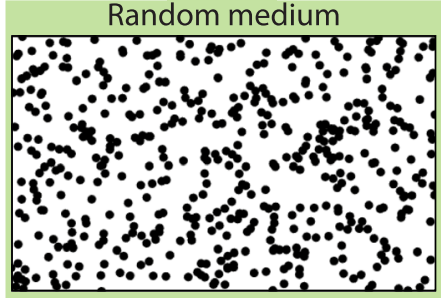

Output wave front $Y$
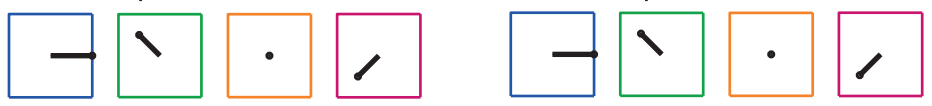

(c)

Simple plane wave front

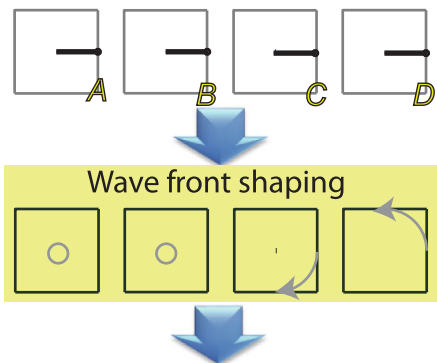

Shaped input wave front

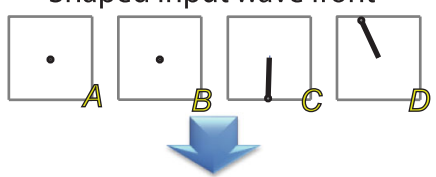

Random medium

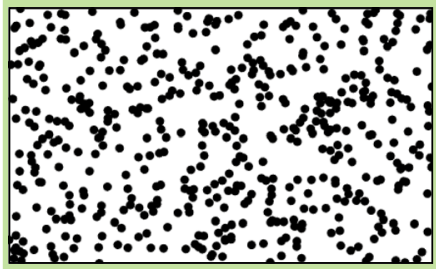

Output wave front $Y$

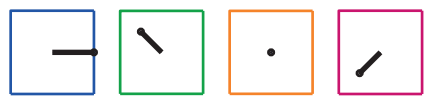

FIG. 1. Schematic comparison of different paradigms for wave-based analog computation (WBAC), illustrated for a $4 \times 4$ discrete

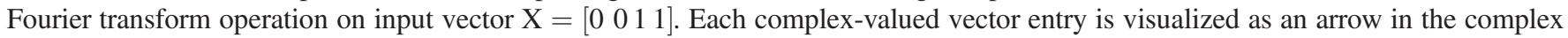
plane. (a) The conventional approach performs the desired operation as the input wave front interacts with a medium of carefully tailored scattering properties. (b) We propose to use any random medium instead of a carefully tailored one-at the cost of appropriately shaping the input wave front before it interacts with the random medium. The proposed WBAC unit thus consists of the wave-front-shaping (WFS) device and the random medium. Each wave-front segment is modulated by several independent pixels of the WFS device, as shown in Fig. 2, acting on the field's phase, as in this schematic, and/or its amplitude. (c) We further suggest a modified version of (b) in which the WFS step additional encodes the desired input vector X into the wave front such that any impinging wave front, e.g., a simple plane wave, can be used. The wave front entering the random medium is then the same as in (b).

implementation of one desired transfer function associated with one desired computational operation $[9,10]$. While conceptually appealing due to the significant miniaturization potential, engineering such a complex structure of meta-atoms in practice with submicrometer tolerances remains a challenge to be mastered [11]. Even slight fabrication imperfections would inhibit the device from performing the desired operation. Moreover, once fabricated, the device could not be reconfigured to perform a different operation.

(iii) Program a coherent nanophotonic circuit to perform a desired operation.-Recent advances in silicon photonics enable the fabrication of a mesh of interconnected Mach-Zehnder interferometers, programmable via phase shifters in the waveguide arms to implement arbitrary linear operations [12-16].
The ability to reconfigure the performed matrixvector multiplication and some first experimental demonstrations reveal the promise held by this emerging research area. Precise beam splitters requiring elaborate fabrication techniques and scalability issues remain critical challenges.

The crux in approaches (ii) and (iii), which enable implementing a desired operation, lies in intricate material designs that are difficult (if not impossible, to date) to fabricate. Here, we propose to overcome any material fabrication and (re)configuration constraints by using a medium with random instead of carefully tailored scattering properties - at the much lower price of appropriately shaping the input wave front before it interacts with the random medium. In principle, our concept preserves all the essential advantages brought about by WBAC, in particular, enhanced computation speed and power efficiency, and 
proposes a way of enjoying them without intricate fabrication procedures. We experimentally demonstrate the validity of our concept in the microwave domain using a chaotic cavity as a random medium and a simple phasebinary metasurface reflect-array to shape the wave field. The fact that we add only a metasurface to a setup emulating standard indoor wireless communication infrastructure shows the ease of implementing our concept in practice, in sharp contrast to any of the current aforementioned WBAC schemes. Then, exploiting the low time cost of WBAC, we demonstrate a scaled-up version under timesequential operation. Finally, we discuss perspectives for practical implementations and how they may compete with traditional electronic processors in terms of speed and energy efficiency.

\section{OPERATION PRINCIPLE}

In the present work, we focus on implementing linear matrix-vector multiplications. As evidenced by substantial industrial efforts to design electronic hardware accelerators (such as ASICs and FPGAs) specialized on such operations, they are a key ingredient of many computational needs. Artificial neural networks capable of deep learning rely on "layers of neurons," each of which consists of a linear matrix-vector multiplication and a nonlinear activation function [17]. The heavy reliance on linear transformations is the computational bottleneck with standard electronic processors. More generally, many key operations in physics and engineering can be expressed as linear operations, such as the discrete Fourier transform that we use as an example in the following. Despite our focus on linear operations, our proposed approach could, in principle, be extended to performing nonlinear operations, for instance, by using nonlinear wave-front-shaping devices [18].

At first sight, the complete scrambling of a wave front as it propagates through a complex medium may seem contrarious to our objective of performing a computation for which a very specific operation is to be carried out on the impinging wave front [19]. Indeed, for a long time, the random secondary sources (scatterers or reflectors) constituting a complex medium were considered undesirable and detrimental. Various novel techniques, notably time reversal and wave-front shaping (WFS), broke that paradigm by leveraging the secondary sources as degrees of freedom (d.o.f.) [20,21]. Inspired by aberration corrections with deformable mirrors in astronomy, WFS applies the appropriate phase and/or amplitude modulation to each segment of an impinging wave front such that, after propagation through the complex medium, the desired output wave front is obtained [21-23]. Initially conceived to counteract the scrambling of a wave front in space and/or time [21,24-27], WFS later on paved the path towards harnessing a medium's complexity, as in the case of focusing beyond the Rayleigh limit achievable in homogeneous media [28-30]. Notably, WFS in complex media enabled the demonstration of programmable beam splitters, the investigation of complex quantum walks as well as custom-tailored mode sorting [31-33]. Moreover, random projections through multiply scattering media were recently shown to be a potentially powerful data preprocessor, for instance, to approximate kernels in machine learning [34].

A linear computation operation may be expressed as a matrix $\mathbf{G}$ that is applied to an input vector $X$, yielding the output vector $\mathrm{Y}=\mathbf{G X}$. To illustrate our general protocol, in Fig. 1(b) we consider the case of a $4 \times 4$ discrete Fourier transform operation for concreteness. We divide the incident wave front into four segments, $A-D$, that will take the role of each of the four entries of the input vector, in our example $X=\left[\begin{array}{llll}0 & 0 & 1 & 1\end{array}\right]$. Each segment has the amplitude and phase of the corresponding input vector entry. Similarly, we observe the wave field after propagation through the medium at four independent points (color coded) that take the role of the output vector's entries. Without any shaping, each wave-front segment yields different random contributions to each of the four observation points. Each of these four wave-front segments covers several independent pixels of the discretized WFS device, depending on the device's discretization, as illustrated in Fig. 2. Then, as shown in Fig. 2(a), each of the pixels has its own random contribution to each of the output points; the overall contribution of segment $D$ is obtained by stringing together the phasors from the contributions of all pixels of which segment $D$ consists, and resembles a random walk in the complex Fresnel representation.

In a one-off initiation step, we first characterize the impact of each pixel of the WFS device on the output wave front, yielding the "impact matrix" (IM) $\mathbf{H}$. The entry $H_{i, j}$ is the complex-valued contribution (amplitude and phase) from the input pixel indexed $i$ to the field observed at the output point indexed $j$. The nature of the IM depends on the system under consideration; in the simplest case of a transmission geometry, as in the schematic in Fig. 1 and standard optical WFS experiments, the IM is simply the system's transmission matrix as in Ref. [24]. The IM provides in all cases an open-loop characterization of the random medium, enabling one to identify numerically specific configurations of the WFS device that satisfy specific objectives. In particular, as shown in Fig. 2(b) for segment $D$, by shaping a multipixel wave-front segment, the corresponding random walks seen in Fig. 2(a) can be converted into tamed walks whose resultants mimic the corresponding entries of G. In other words, WFS enables one to force the contribution from each input entry to each output entry to be exactly the one required by the desired operation G. Notice the key role played by the random medium by enabling each input segment to contribute to each output point - if the random medium was replaced by free space, only diagonal matrices $\mathbf{G}$ could be implemented. The shaped wave front for segment $D$ has to simultaneously yield contributions with specific phases and 


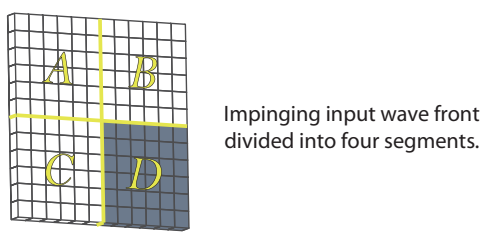

(a) Contribution of segment $D$ to observation points

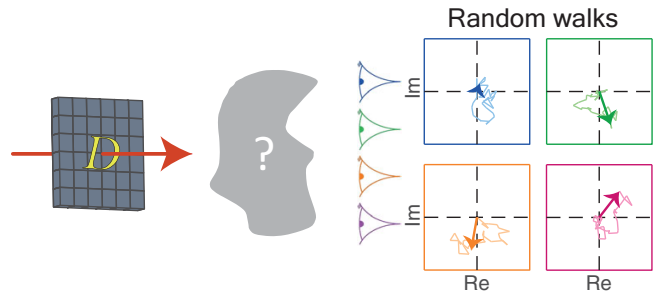

(b) Tamed contribution of segment $D$ to observation points

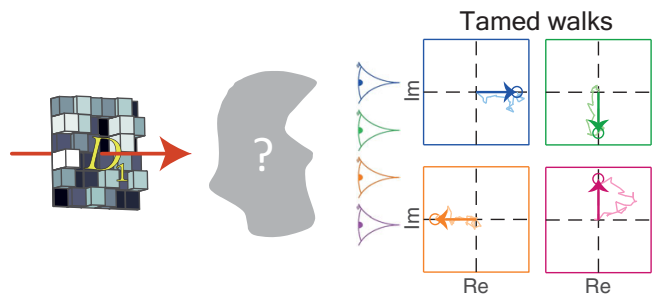

FIG. 2. The impinging input wave front in Fig. 1(b) consists of four segments taking the role of a four-element input vector. This figure illustrates the contribution of one of these segments, $D$, to all four observation points after propagation through the complex medium, contrasting instances without (a) and with (b) wave-front shaping of the impinging wave front. Each segment consists of $n_{\text {seg }}$ pixels, corresponding to a discretized wave-front-shaping device, with $n_{\text {seg }} \geq 1$ in general. (a) A plane wave entering a complex medium gets completely scrambled. At each observation point, stringing together each pixel's contribution yields a random walk whose resultant (the collective contribution from segment $D$ ) is indicated by a bold arrow. (b) The discretized wave front of segment $D$ can be shaped such that, after propagation through the complex medium, the resultants at each observation point mimic the entries of the corresponding row of $\mathbf{G}$. The latter are indicated by circles; for our example of a discrete Fourier transform, the fourth row of $\mathbf{G}$ reads $[1-i-1 i]$.

amplitudes to all four observation points, a constraint that is much harder to satisfy than simple focusing, which requires only aligning the phasors in an arbitrary angular direction at a single observation point. Moreover, note that we do not tame the entire field at the observation points but the contributions from specific wave-front segments to the field at the observation points. Identifying the appropriate configurations for each segment of the WFS device based on the measured IM is the second step of the initiation procedure, and it is performed purely numerically. A key aspect about this initiation procedure is that to perform a different operation $\mathbf{G}$ one does not require any further measurements; one simply goes through the numerical optimization once again.

Once a library of optimized configurations for each wave-front segment is identified, an impinging wave front can be shaped such that its subsequent propagation through the complex medium carries out the desired computational task at the speed of wave propagation, as summarized in Fig. 1(b). This concept shifts the burden from designing and fabricating the physical layer's scattering properties to identifying and imposing appropriate wave fronts, which is substantially simpler in terms of both the optimization problem as well as the experimental implementation. Instead of encoding the input vector $\mathrm{X}$ into the impinging wave front, alternatively, X may be encoded directly into the configuration of the WFS device such that a plane impinging wave front can be used. This idea is visualized in Fig. 1(c). The underlying thought is that, most likely, in order to have the input vector encoded into the impinging wave front, some WFS device outside the considered WBAC system is employed. Then, however, instead of using essentially two WFS devices directly one after the other, one could use a single WFS device to do both tasks. In Fig. 1(c), we show how the WFS step simultaneously encodes the input into the impinging plane wave front and adds the modulation required given the random medium to perform the desired operation. Then, the wave front entering the random medium is the same as in Fig. 1(b).

\section{DEMONSTRATION WITH INDOOR WIRELESS COMMUNICATION SIGNALS}

Having outlined the general principle of operation, we now proceed with an experimental demonstration that seeks to stress (i) the ease of implementation in contrast to hitherto existing WBAC proposals and (ii) the wide applicability to all types of waves and random media. The first complex medium that comes to mind is usually a multiply scattering one. In the microwave domain, examples thereof exist, such as cities or forests, but they are highly unpractical for experiments. Instead, cavities of irregular geometry lend themselves to practical, wellcontrolled, and stable setups and are, moreover, very commonplace. Indeed, indoor environments constitute cavities of low quality factor for the microwaves used in wireless communication protocols such as Wi-Fi. Their geometries are, in general, rather irregular such that these cavities may be labeled "random" or "chaotic" from the wave's perspective. The reflections off the irregular cavity walls interfere and give rise to a specklelike wave field [35], very much like scattering events in multiply scattering optical media such as thin paint layers or biological tissue [21,36]. Furthermore, complex microwave cavities are leveraged in fundamental research on quantum chaos [37] as well as in applications ranging from security screening [38,39] and biomedical imaging [40], via sensing 
[41,42] and wireless power transfer [43-46] to electromagnetic compatibility tests [47].

\section{A. Shaping cavity Green's functions}

Despite the conceptual similarity between multiply scattering media and chaotic cavities, there are important nuances that we discuss here to motivate our subsequent course of action. A convenient way of probing the field in a cavity is to measure the Green's function between two independent positions [48-51]. To get a firmer grasp of a chaotic cavity's physics, it is instructive to express the transmitted field between two antennas in terms of the modal contributions. Omitting for clarity both details of the antenna coupling as well as the vector nature of the electromagnetic field, we can write the Green's function $S$ between two points $r_{i}$ and $r_{j}, r_{i} \neq r_{j}$, as

$$
S\left(\mathrm{r}_{\mathrm{i}}, \mathrm{r}_{\mathrm{j}}, f_{0}\right) \propto \sum_{n=1}^{N} \frac{\psi_{n}\left(\mathrm{r}_{\mathrm{i}}\right) \psi_{n}\left(\mathrm{r}_{\mathrm{j}}\right)}{\frac{4 \pi^{2}}{c^{2}}\left(f_{0}^{2}-f_{n}^{2}\right)+2 \pi i f_{0} \Gamma},
$$

where $\psi_{n}$ and $f_{n}$ are the eigenvector and eigenvalue, respectively, of the $n$th cavity mode that contributes to the Green's function at the working frequency $f_{0}$. $\Gamma$ is the (average) modal linewidth. An estimate of the number of modes $N$ overlapping at $f_{0}$, this time accounting for the three-dimensional and polarized nature of the field, can be obtained from Weyl's law [52-54]:

$$
N=\frac{8 \pi f_{0}^{3}}{c^{3}} \frac{\mathcal{V}}{Q}
$$

$\mathcal{V}$ and $Q$ being the cavity's volume and quality factor, respectively. In a chaotic cavity, $\psi_{n}$ can be modeled as a random variable such that $S\left(\mathrm{r}_{\mathrm{i}}, \mathrm{r}_{\mathrm{j}}, f_{0}\right)$ can be interpreted as a random walk of $N$ steps in the complex plane-just as illustrated in Fig. 2(a).

The crucial remaining question is: How can a cavity Green's function be shaped? The concept of WFS in complex media originally emerged in optics [21] but has recently been transposed to the microwave domain using tunable metasurface reflect-arrays, with important applications in telecommunication, imaging, sensing, and energy transfer [39-42,45,46,55,56]. Different designs of such reconfigurable metasurfaces are discussed in the literature [39,57-61]; here, we use a simple phase-binary device whose working principle is based on the hybridization of resonances (see Ref. [60] and Sec. A in Ref. [62] for further details). We can electronically configure the phase shift of the reflected wave for each of the 88 metasurface elements to be 0 or $\pi$. Stated differently, each metasurface element can be programmed to mimic a Dirichlet or a Neumann boundary condition.

By partially altering the cavity's boundary conditions with the tunable metasurface, we have some control over the modal sum in Eq. (1), because we essentially modify the cavity eigenmodes $\psi_{n}$. The degree of control depends both on the cavity's modal overlap which fixes $N$ [see Eq. (2)] and on the amount of metasurface elements, yielding the capability of "effectively" controlling $p$ out of the $N$ modes that contribute to a cavity Green's function at the working frequency [54]. As discussed in Ref. [54], the ratio $p / N$ defines distinct regimes: (i) If $N>p$, we control effectively a subset of the contributing modes such that the random walk can be tamed partially; (ii) if $N \approx p$, we have full control over the modal sum and can tame the walk at wish; (iii) if $N \ll 1<p$, the cavity spectrum consists of discrete resonances that can be created and shifted at will.

\section{B. Choosing a convenient regime}

Unlike the simple transmission geometries used in Sec. II to illustrate our WBAC scheme, the physics of a chaotic cavity is richer than captured by a simple, linear transmission formalism. Here, the IM captures the impact of each metasurface reflect-array pixel on a selection of Green's function measurements. Since the reverberating wave revisits each pixel of the WFS device multiple times, the impact of a given pixel on the wave field is correlated to some extent to the configuration of the remaining pixels: $\mathbf{H}$ is itself a function of the metasurface reflect-array configuration. A pixel acts not like a single but multiple (secondary) sources, due to reverberation.

In principle, the realm of machine learning with artificial neural networks [17] offers elegant ways of accurately capturing the full IM including the long-range correlations. However, having such a complete forward model would not alter the fact that physically the contributions of a given "input" group of pixels on the WFS device to the "output" Green's function measurements depend to some extent on how the remaining pixels of the WFS device are currently configured. In other words, in a cavity, one cannot hope to be able to shape the contributions of one wave-front segment independently from the other ones. What appears at first sight to be an insurmountable hurdle can, in fact, be easily resolved by leveraging the cavity's chaotic nature.

First, we deliberately measure only a first-order approximation of $\mathbf{H}$ that ignores the long-range correlations. That is, we neglect the dependence of a pixel's wave field impact on the configuration of the remaining pixels, yielding a linear forward model. Second, we numerically identify a library of segment configurations that appropriately tame the wave front for the desired computational operation (see Sec. III D for the detailed numerical procedure), supposing our first-order IM is exact. Of course, the computation outcomes that we expect based on our approximated IM, $\mathrm{Y}^{\text {pred }}$, are not exactly what we measure, $\mathrm{Y}^{\text {meas }}$, since we do not account for the long-range correlations yet. The effect of the latter on the $j$ th entry of the computation output can be interpreted as a random phasor $\delta_{j}: Y_{j}^{\text {meas }}=Y_{j}^{\text {pred }}+\delta_{j}$. For a given realization, $\delta_{j}$ is a deterministic but seemingly 
arbitrary random complex number that results in an inevitable inaccuracy of our computation outcome $Y_{j}^{\text {meas }}$. A realization may be defined as one instance of the experiment with some specific (irregular) cavity geometry. A different realization has the same global parameters (cavity volume, quality factor, etc.) but a different geometry and, thus, a different random $\delta_{j}$. Therefore, we can exploit the realization dependence of $\delta_{j}$ to average out the intrinsic inaccuracy: $\left\langle\delta_{j}\right\rangle_{\text {realizations }}=0$, and, thus, $\left\langle Y^{\text {meas }}\right\rangle_{\text {realizations }}=Y^{\text {pred }}$. By ensemble averaging over different realizations, we are thus able to mitigate the adverse effects of long-range correlations in the IM.

The importance of long-range correlations on the impact of pixel $i$ on the transmission measurement indexed $j$ is defined by two parameters that govern how likely it is that a ray that interacts with pixel $i$ also encounters other pixels during its lifetime: (i) The higher $Q$ is, the longer the ray's lifetime is, and the more pixels other than $i$ it encounters; (ii) the higher the number of pixels, the easier it is for the ray to encounter some of the other pixels. It is instructive to think of an IM entry $H_{i, j}$ as consisting of static contributions from rays that interacted only with pixel $i$ and of variable contributions from rays that interacted also with other pixels of the metasurface reflect-array. Ultimately, we average out the effect of the latter such that our approach works as long as the former is nonzero. The impact of a given pixel of the WFS device on the wave field must not completely depend on how the other pixels are configured.

An ideal fit in sight of these considerations is thus operating in the regime $N>p$ in an electrically large cavity of (relatively) low $Q$. Of course, reverberation is crucial to ensure the cavity acts like a random medium, but limiting $Q$ to the order of $10^{2}$ rather than $10^{3}$ or $10^{4}$ addresses (i). Simultaneously, since $N$ is large under these conditions [see Eq. (2)], one can fit a large metasurface with many pixels into the cavity and still respect (ii). One might speculate that, as $N$ becomes larger than $p$, the metasurface completely dominates the wave field and the static cavity contribution to $H_{i, j}$ vanishes-ultimately, confirming this speculation remains a topic for future work. It is clear, however, that the limiting case of very-high- $Q$, smallvolume cavities in the regime $N \ll 1<p$ with discrete resonances is inappropriate for our scheme [54,63,64].

\section{Experimental setup}

In sight of the above considerations, our experimental setup thus consists of an irregularly shaped cavity with a (relatively) low quality factor and the ability to access different realizations for ensemble averaging. In order to operate under stable, well-controlled conditions, we work in a metallic cavity of irregular geometry $(\mathcal{V}=1.01 \times$ $\left.0.86 \times 1.28 \mathrm{~m}^{3}=1.1 \mathrm{~m}^{3}\right)$. By placing absorbers on its walls, we limit to some extent the reverberation with the previously outlined motivation; the resulting quality factor
$Q=179$ is comparable to the one we measure in an office room. Given our working frequency $f_{0}=2.65 \mathrm{GHz}$, there are thus $N \approx 106$ overlapping modes. Our metasurface consisting of $n=88$ elements can effectively control about $p \approx 2 \times(n / 3)=59$ of these modes (see Sec. A in Ref. [62]). We are thus operating in the first regime where $N>p$, under conditions that are typical for an indoor environment. A schematic is shown in Fig. 3. Figure $S 1$ in Ref. [62] illustrates with experimental data that in our setup $H_{i, j}$ is indeed dominated by the static cavity contribution, as desired.

We need as many independent single-frequency transmission measurements as our output vector $\mathrm{Y}$ is supposed to have entries. We choose to place antennas at arbitrary positions inside the cavity that are at least half a wavelength apart, which is an obvious option given the wave field's specklelike nature with a correlation length on the order of half a wavelength; alternatively, one may also use a single pair of antennas and multiple working frequencies that are sufficiently different [65]. In the realistic scenario of indoor wireless communication systems, the channel state information (CSI) between an access point and a wireless device

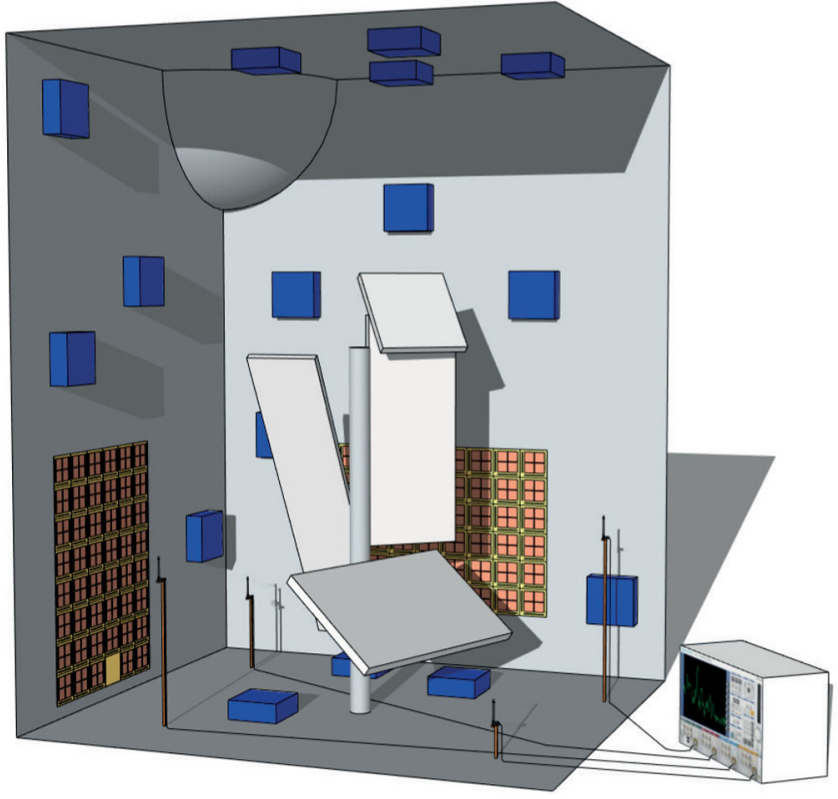

FIG. 3. Laboratory setup to test the feasibility of analog computation with Wi-Fi waves in an indoor room. About $6 \%$ of a chaotic metallic cavity's surface are covered with a metasurface reflect-array consisting of 88 pixels with a tunable reflection coefficient ( $r= \pm 1)$; see Ref. [60] and Sec. A in Ref. [62] for the detailed working principle. Electromagnetic absorbers placed on the walls lower the cavity quality factor to $Q=179$, comparable to an indoor room. With a vector network analyzer, we measure the transmission between four simple monopole Wi-Fi antennas, mimicking channel state information between an access point and four mobile devices. A mode stirrer, rotated in steps of $12^{\circ}$, enables ensemble averaging over realizations of the experiment under different (irregular) cavity geometries. 
is exactly what we need: a complex-valued transmission measurement between two points inside the cavity. Modern multiuser telecommunication networks routinely sound channels, for instance, using beacon signals, for down-link beam forming [66]. CSI can be accessed with off-the-shelf Intel 5300 cards and has successfully been extracted and used, for instance, as a fingerprint for object localization [67]. Green's function measurements for different pairs of $r_{i}$ and $r_{j}$ are thus readily available in commonplace wireless communication infrastructure. In our laboratory implementation, we mimic CSI between wireless devices and a base station by transmission measurements between independent monopole antennas with a vector network analyzer.

Ensemble averaging is easily achieved in our experiment, since we can access a new realization either by rotating a mode stirrer by $12^{\circ}$ (see Fig. 3 ) or by changing the operating frequency by more than a correlation frequency (see Sec. A in Ref. [62]). Both concepts could, of course, be used in an analogous manner with Wi-Fi in an indoor room. The constant motion of inhabitants naturally provides new realizations by physically changing the cavity's irregular geometry, analogous to the mode stirrer in our experiment. A single realization would have to be carried out within the coherence time of the medium, which is within reach in sight of real-time switching of the $p-i-n$ diodes controlling the reflect-array with improved electronics [68]. Alternatively, using the CSI between different pairs of access points and mobile devices (e.g., if there are many mobile devices in a room) is another way to obtain independent realizations of the experiment without relying on motion inside the room.

\section{Experimental procedure}

Measuring the impact matrix (IM).-The entry $H_{i, j}$ quantifies the impact of the reflect-array element indexed $i$ on the transmission between the pair of antennas indexed $j$. We measure the IM using the Hadamard rather than the canonical basis, following the procedure used in Ref. [24]. The elements of the Hadamard basis being +1 or -1 , this is a perfect match to our experiment where we can access exactly these two values for each pixel's reflection coefficient. Moreover, working in the Hadamard basis has a favorable averaging effect as illustrated in Fig. S1 in Ref. [62] and benefits furthermore from an improved signal-to-noise ratio.

One important difference to Ref. [24], however, is that we control only a portion of the wave field. As elaborated in the previous section, we operate in the first regime where $N>p$, and we effectively control only about $p / N \approx 56 \%$ of the cavity modes. To successfully define an IM, we subtract from each transmission measurement $Y_{j}$ the static cavity contribution $U_{j}$ that cannot be altered by the metasurface. This static contribution corresponds to the uncontrolled cavity modes, which, hence, resist averaging over random reflect-array configurations. We thus obtain
$U_{j}$ as $\left\langle Y_{j}\right\rangle_{\text {random } \mathrm{V}}$, where $\mathrm{V}$ is a vector defining the reflectarray configuration, and then we work with $\mathrm{Y}^{\prime}=\mathbf{H V}$, where $\mathrm{Y}^{\prime}=\mathrm{Y}-\mathrm{U}$ as in Ref. [69]. Note that this subtraction is necessary only for a cavity implementation of our proposed WBAC scheme; in transmission geometries as in Fig. 1, there is typically no uncontrolled contribution to the output wave front in any case, since the entire incident wave front interacts with the WFS device.

Numerical identification of wave-front segment configurations.-Given the IM, we numerically identify the reflect-array configurations for each wave-front segment that yield the desired tamed walks illustrated in Fig. 2(b). Consider for concreteness once again the $4 \times 4$ example given in Figs. 1 and 2. Let $\mathrm{V}_{D}$ be the part of vector $\mathrm{V}$ that belongs to segment $D$, let $Y_{j}^{D}$ denote the contribution of segment $D$ to the measurement $Y_{j}$ at observation point $j$ (that is, $Y_{j}^{D}$ is a single complex-valued number), and let $\mathrm{H}_{D, j}$ be the corresponding part of the IM $\mathbf{H}$ that links $\mathrm{V}_{D}$ to $Y_{j}^{D}$. In summary, $Y_{j}^{D}=\mathrm{H}_{D, j} \mathrm{~V}_{D}$. Note that selecting the members of segment $D$ among all pixels of the WFS device does not have to be done in a regular manner as in our schematic in Fig. 2. In our experiment, we make the selection randomly; a careful selection could, in fact, be a further parameter of the optimization. The objective is to find a $\mathrm{V}_{D}$, given $\mathrm{H}_{D, 1}$ to $\mathrm{H}_{D, 4}$, such that simultaneously specific values of $Y_{1}^{D}$ to $Y_{4}^{D}$ are obtained. This objective can be expressed as a single stacked equation: $\left[\mathrm{H}_{D, 1}, \mathrm{H}_{D, 2}\right.$, $\left.\mathrm{H}_{D, 3}, \mathrm{H}_{D, 4}\right] \mathrm{V}_{D}=\left[Y_{1}^{D}, Y_{2}^{D}, Y_{3}^{D}, Y_{4}^{D}\right]$. In the case of perfect phase and amplitude control over each pixel of the WFS device, the equation is easily solved by calculating the pseudoinverse of $\left[\mathrm{H}_{D, 1}, \mathrm{H}_{D, 2}, \mathrm{H}_{D, 3}, \mathrm{H}_{D, 4}\right]$-provided each wave-front segment is assigned a sufficient number of pixels of the WFS device. Indeed, from basic considerations about the singular value decomposition of this stacked matrix, it becomes clear that we need to assign at least $\mathcal{N}$ idealistic pixels to each wave-front segment in order to be able to calculate a meaningful pseudoinverse [70]. In our experimental setup, however, the entries of $\mathrm{V}$ may take only the values of +1 or -1 due to our phasebinary-only metasurface control over the wave front. A plethora of methods exist to solve the ill-posed problem resulting from this constraint [71]. Here, we opt for a homemade solver inspired by standard iterative sequential optimization algorithms used in optical WFS [72].

First, we define a normalization factor $\gamma$ between the entries of $\mathbf{G}$ and the target amplitudes of the tamed walks. We choose $\gamma=n_{\text {seg }}^{1 / 2}\left\langle\left|H_{i, j}\right|\right\rangle_{i, j}$, where $n_{\text {seg }}$ is the number of pixels in a given wave-front segment. This choice corresponds to the expected value of the resultant of a random walk of $n_{\text {seg }}$ steps of step size $\left\langle\left|H_{i, j}\right|\right\rangle_{i, j}$. Next, we define a cost function as $\mathcal{C}=\left\langle\left|\gamma G_{D, j}-\mathrm{H}_{D, j} \mathrm{~V}_{D}\right|\right\rangle_{j}$. $\mathcal{C}$ is thus the distance in the complex plane between an entry of $\gamma \mathbf{G}$, say, the entry linking input segment $D$ to observation point $j$, $\gamma G_{D, j}$, and the corresponding value of $\mathrm{H}_{D, j} \mathrm{~V}_{D}$, averaged 
over all output points $j$. Starting with a random configuration of $\mathrm{V}_{D}$, we test element by element if flipping its state reduces $\mathcal{C}$, in which case we update $\mathrm{V}_{D}$ accordingly. We run 20 loops over all elements. Finally, we repeat the procedure with 250 different random initial $\mathrm{V}_{D}$ and select the overall best final $\mathrm{V}_{D}$ yielding the lowest $\mathcal{C}$ as an entry for the library. Note that this numerical procedure is not heavily optimized, and more efficient schemes could certainly be found $[73,74]$. In any case, whatever the numerical cost to identify the wave-front segment configurations, this numerical optimization procedure is a one-off effort during the initiation phase of our WBAC scheme.

Given the peculiarity of our chosen cavity implementation in which the WFS device is essentially inside the propagation medium and its pixels are secondary sources, it is convenient and most meaningful to adopt the WBAC scheme from Fig. 1(c), in which the WFS device additionally encodes the input vector $\mathrm{X}$. In principle, additionally encoding the input is a trivial step. However, in our experiment, since we do not have perfect phase and amplitude control on the reflect-array, this step is not straightforward. To encode an input vector entry $\alpha$ other than unity into, e.g., $\mathrm{V}_{D}$, we thus identify a new configuration for $\mathrm{V}_{D}$ such that its contribution $\mathrm{H}_{D, j} \mathrm{~V}_{D}$ to the observation point indexed $j$ is $\alpha \gamma G_{D, j}$, simultaneously for all $j$. In this section, we restrict ourselves to $\alpha \in\{0,1\}$, which corresponds to a 1-bit input resolution. We consider a larger input size and resolution in Sec. III F.

\section{E. Experimental results}

In Figs. 4(a)-4(d), we present our experimentally obtained computation results $\mathrm{Y}$ for the discrete Fourier transform operation on four different input vectors $X$, based on an ensemble average of $\mathrm{Y}^{\text {meas }}$ over 150 realizations (30 mode-stirrer positions and five independent working frequencies). We observe an excellent agreement with the theoretically expected results, the average computation error $\epsilon=\left\langle\left|Y_{j}^{\text {exact }}-Y_{j}^{\text {meas }}\right|\right\rangle_{j}$ being $3.8 \%$ (averaged over all possible input vectors $\mathrm{X}$ ).

The dependence of $\epsilon$ on the number of realizations displayed in Fig. 4(e) confirms that the inaccuracy due to (consciously) neglecting the long-range correlations in the IM can indeed be averaged out successfully by ensemble averaging over different realizations. A further illustration of this effect based on experimental data is provided in Fig. S2 of Ref. [62].

Incidentally, we note that ensemble averaging is also extremely useful in the case of very limited wave-front control. For instance, in our experiment, each of the four wave-front segments is made up of only 22 phase-binary pixels. As seen by the purple curve in Fig. 4(e), 22 phasebinary pixels provide on average not enough control to perfectly tame the contributions from all input segments to all observation points. For a single realization, an entry of the

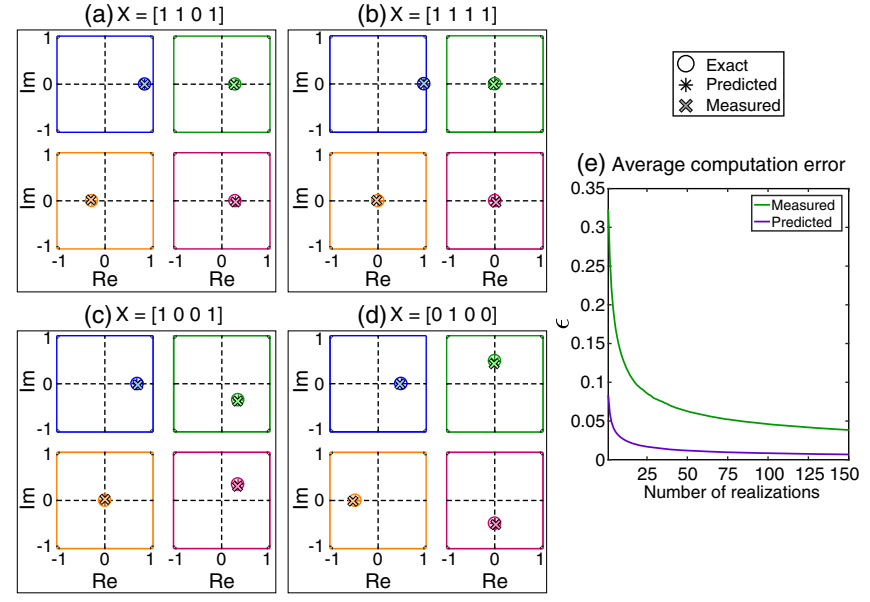

FIG. 4. Experimentally obtained computation results for the complex-valued $4 \times 4$ discrete Fourier transform operation. (a)-(d) Results for the different indicated input vectors $X$, normalized such that $\Sigma_{j}\left|Y_{j}\right|^{2}=1$ for easy comparison. The experimentally measured values $\mathrm{Y}^{\text {meas }}$ (cross symbol) are shown together with the theoretically expected results for $\mathrm{Y}=\mathbf{G X}$, $\mathrm{Y}^{\text {exact }}$ (circle symbol), as well as the results predicted by the measured IM, Y Yred (star symbol); cf. the legend. (e) Dependence of the average computation error $\epsilon=\langle| Y_{j}^{\text {exact }}-$ $Y_{j}^{\text {meas }}|\rangle_{j}$ (green line), averaged over all possible $\mathrm{X}$, on the number of realizations over which the computation outcome is averaged. We contrast $\epsilon$ with the average predicted error $\epsilon^{\prime}=\langle| Y_{j}^{\text {exact }}-$ $Y_{j}^{\text {pred }}|\rangle_{j}$ (purple line), where $\mathrm{Y}^{\text {pred }}$ is the expected computation outcome assuming the IM is exact.

predicted computation outcome $Y_{j}^{\text {pred }}$, which does not suffer from the long-range correlations since it assumes the IM is exact, is not exactly equal to the exact result $Y_{j}^{\text {exact }}$ expected for the operation. Once again, we can interpret this effect of imperfect wave-front taming as a random realization-dependent phasor $\delta_{j}^{\prime}: Y_{j}^{\text {pred }}=Y_{j}^{\text {exact }}+\delta_{j}^{\prime}$. By the same token as before, $\left\langle\delta_{j}^{\prime}\right\rangle_{\text {realizations }}=0$; indeed, as shown by the purple line in Fig. 4(e), $\epsilon^{\prime}=\left\langle\left|Y_{j}^{\text {exact }}-Y_{j}^{\text {pred }}\right|\right\rangle_{j}$ is reduced from $8.3 \%$ to $0.7 \%$ by ensemble averaging. Hence, the idea of ensemble averaging serves two purposes in our case.

The presented results not only constitute a proof-ofprinciple experimental implementation of our proposed scheme for WBAC with a random medium but allow us, moreover, to conclude that it takes nothing but standard indoor wireless communication infrastructure and a simple tunable metasurface to perform accurate analog computation with reverberating microwaves. While we could have used any complex-valued matrix for our demonstration, we deliberately selected the (discrete) Fourier transform operation. Besides its key role in all areas of physics and engineering, it also facilitates interesting interpretations of our experimental results. Complex media in conjunction with WFS in optics, or time reversal in acoustics, have repeatedly been described as "opaque lens" or "scattering lens" in the literature $[22,75]$, alluding to the most basic 
property of a traditional lens: It focuses light in geometrical optics. In our present work, we use a complex medium to Fourier transform an impinging wave front such that it becomes possible to extend the analogy from the ray picture to the properties of a traditional lens in the realm of "wave" optics. While a lens in optics is a standard component, transposed to the microwave domain, our concept unveils yet more of its intriguing properties: (i) Lenses are not as widely used; (ii) reflect-arrays and antennas, mimicking the input and output of the Fourier transform, are not aligned in any way but placed randomly, and the medium carrying out the operation is around rather than between inputs and outputs. Essentially, by configuring the reflect-array according to the previously established library for a given input $\mathrm{X}$, we create a cavity geometry in which the transmission between the selected antenna pairs corresponds to the computation output Y.

\section{F. Scalability of resolution and operation size}

Typical concerns with WBAC schemes include questions about the achievable resolution and system size. In the realm of artificial neural networks (ANNs) that constitutes as aforementioned an important potential field of application for WBAC, limited resolution is, in fact, by no means an insurmountable barrier. The accuracy loss of 8-bit artificial neural networks in comparison with 32-bit computers was shown to be only $0.4 \%$ on standard tasks [76]; moreover, if the ANN has been specifically designed for low resolution, it can achieve similar results with as little as 1-bit or 2-bit resolution.

If we were to bluntly apply our previous procedure with the same physical system to a significantly larger computational operation, identifying appropriate configurations for each segment would become doubly more difficult: (i) Given the fixed reflect-array size, each wave-front segment would consist of even fewer pixels, further reducing the available d.o.f.; (ii) each segment configuration would have to simultaneously satisfy even more constraints, since there are also more observation points due to the larger size of the output vector Y. Averaging over more realizations could cushion these adverse effects to some extent, but there are more elegant solutions.

In the following, we extend our previous experiment with 1-bit input resolution and 4-entry input size to a system with more than 4-bit input resolution and a 16-entry input size-using exactly the same physical system. To access a better resolution, we simply perform the numerical optimization step outlined in Sec. III D for a larger range of values of $\alpha$. Note that these additional optimization steps are necessary only because of the phase-binary-only control of our metasurface reflect-array. To access a larger system size, we exploit the extremely low time cost of WBAC. Indeed, since the computation in our cavity implementation is carried out as the stationary wave field is established, the only potentially limiting factors are the field's transient duration and the duration of the measurement itself that is required to read off the computation result. We simply break our computational task down into several smaller ones that fit our physical system's size. For ANNs, rather than processing an input signal such as an image instantaneously, it has already become common practice to feed the input into the system via multiple patches one after the other-even without the benefit of ultrafast computation times as in WBAC [77]. Here, we demonstrate a time-sequential WBAC version that exploits the linearity of the computation operation $\mathrm{Y}=\mathbf{G X}$ in which the entries of $\mathrm{Y}$ are independent from each other. Thereby, we compute one entry of Y after the other such that we convert one $\mathcal{N} \times \mathcal{N}$ operation into $\mathcal{N} 1 \times \mathcal{N}$ operations (see Fig. S3 in Ref. [62] for an illustration) that are performed one after the other. This procedure is possible thanks to the reconfigurability of our wave-based analog computation unit - a fundamental advantage of our proposed system over metamaterial computation units fabricated for a single specific task.

In principle, this idea enables the computation of a subset of output entries simultaneously, in the following one at a time, depending on the size of the desired operation and the size of the available physical system. Even if we compute "only" one output entry at a time, the random medium still plays a crucial role in mixing all the tamed contributions from the different input segments to that single output observation point; the numerical optimization of wave-front segment configurations during the one-off initiation step of our WBAC scheme is still more demanding than a simple focusing experiment, since we still need to accurately mimic one entire row of $\mathbf{G}$. In other words, we still need to accurately tame as many random walks as there are input entries rather than only aligning the steps of a single walk.

We present in Fig. 5 the experimentally obtained Fourier transforms of the silhouettes of two Parisian monuments. To faithfully reproduce the silhouettes, we used an input resolution of more than 4 bits, clearly superior to the 1-bit resolution from Fig. 4. We once again ensemble averaged over 150 realizations of this $16 \times 16$ complex-valued computation (see Sec. D in Ref. [62] for details on G and further examples). The obtained results are in good agreement with the exact ones, showing that the Eiffel Tower creates a richer spectrum than Notre Dame, since it has more structural details.

Note that the presented multiplexing in time could, in principle, also be implemented in space with $\mathcal{N}$ identical WBAC units. This alternative implementation might be of interest for optical hardware accelerators to circumvent a potential upper limit on computation power per system (limited d.o.f. in a complex medium or limited number of pixels on the WFS device) to maintain the ultrafast computation time.

WBAC also offers further exciting opportunities for parallelization of computational tasks. One can, for 


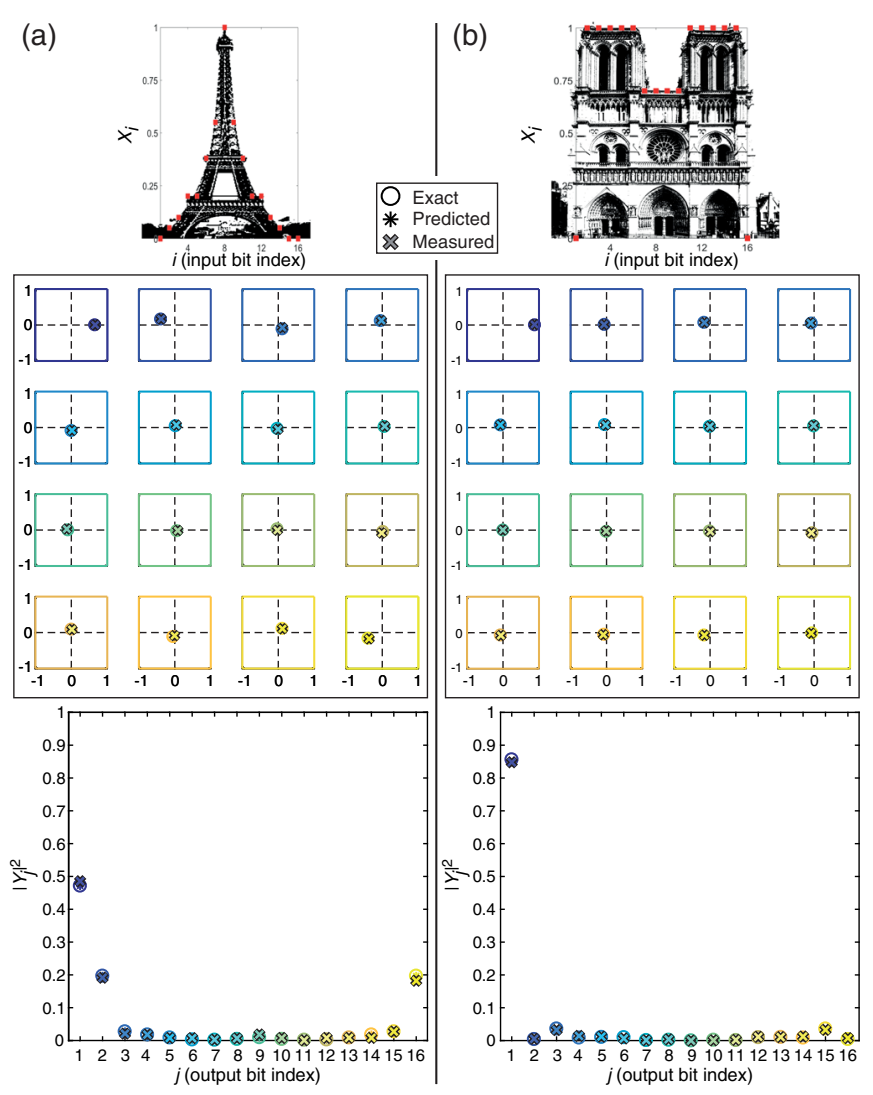

FIG. 5. Demonstration of sequential wave-based analog computation. We reconfigure the computation unit to compute one output entry at a time, which allows us to tackle larger computational tasks. The figure shows the computed Fourier transforms of the silhouettes of two Parisian monuments: (a) the Eiffel Tower and (b) Notre Dame. We display the experimentally obtained results in the complex plane as well as in terms of the intensities. The exact results are also indicated; see the legend.

instance, imagine to operate our scheme at two or more clearly distinct working frequencies within the same cavity equipped with one metasurface per working frequency, each metasurface operating within a narrow band around its corresponding working frequency. At a given working frequency, the other metasurfaces not operating at that working frequency would simply act like a metal wall without any modulating effect.

\section{DISCUSSION AND OUTLOOK}

Practical, stable, and efficient cavity implementation.The size of the discussed cavity implementation can conveniently be scaled down by operating at a higher frequency. For example, operating in the K-band around $20 \mathrm{GHz}$ would reduce the volume of the previous experiment by a factor of $10^{-3}$, yielding cavity dimensions that can potentially even be integrated into data-center racks. Mode stirring can conveniently be performed in an allelectronic manner, by adding more metasurface pixels dedicated to mode stirring; putting these pixels into different random configurations yields different realizations $[78,79]$. This approach provides very fast access to more independent configurations than a mechanical mode stirrer. The different realizations over which the computation outcomes are averaged can be predefined such that all initiation steps are performed before the actual computation phase. The metasurface design can be upgraded, for instance, for linear phase control with a varactor-populated mushroom structure as in Ref. [39]. The cavity constitutes a very stable enclosure that is completely shielded from the outside. The stability implies that, once measured, the IM would not lose validity over time. The shielding allows for a fantastic signal-to-noise ratio. The very low noise level almost eliminates any limits on resolution caused by effective truncation errors originating from noise. Moreover, the low noise level is very favorable for highspeed low-power operation, as discussed below.

Speed.-The fundamental advantage in operation speed over traditional processors originates from the fact that a WBAC unit performs a task in one go that requires many consecutive operations on a digital computer. For instance, an $\mathcal{N} \times \mathcal{N}$ matrix multiplication that we perform in a single run requires, in general, $O\left(\mathcal{N}^{2}\right)$ digital operations. Traditional computers, having clock rates on the order of $1 \mathrm{GHz}$, can perform $\sim 10^{9}$ operations per second. In our cavity scheme, the limiting factor is the time it takes for the wave field to reach equilibrium, on the order of $\tau \sim 1 / \Delta f_{\text {corr }} \sim 5 \mathrm{~ns}$ for the K-band version, since measurements even at gigahertz rates are possible with standard technology - aided by the low-noise nature of the shielded cavity. Accounting for the need for ensemble averaging, we think one entire computation can be performed on the order of $1 \mu \mathrm{s}$. The actual averaging over realizations could be performed directly on the detector, similarly to a camera that integrates the incident light over desired time intervals. Subtracting the constant cavity contribution $U_{j}$ from each observation point, given prior calibration, could probably be integrated into the measurement process, too, but in any case this is only of $O(\mathcal{N})$ complexity. Thus, we expect to be able to perform the equivalent of $\mathcal{N}^{2} \times 10^{6}$ digital operations per second. Clearly, the larger $\mathcal{N}$ gets, the higher is the advantage of the WBAC scheme. The break even is expected for operation sizes on the order of $\mathcal{N} \sim 30$.

Energy efficiency.-A traditional computer uses at least $100 \mathrm{pJ}$ per operation $[15,80]$. The power consumed by our cavity WBAC scheme is dominated by the feeding circuits of the metasurface pixels: $25 \mu \mathrm{W}$ per pixel (see Ref. [55] and Sec. E in Ref. [62] for further details). This number could be reduced by taking power consumption as one of the design criteria of the metasurface. Using $\mathcal{N}^{2} \times 10^{-6} \mathrm{~W}$ as the power needed to perform an $\mathcal{N} \times \mathcal{N}$ operation then yields an estimated energy consumption of $1 \mathrm{pJ}$ per equivalent digital operation. Thus, the scheme has the potential to compete with or even outperform a traditional computer in terms of energy efficiency. 
Perspectives in optics. - An optical implementation of our scheme in a transmission geometry as in Fig. 1(b) holds several promises, notably that the IM would not have any long-range correlations (and thus no intrinsic need for ensemble averaging), the operation speed in a transmission geometry would be limited only by the detection rate, which could be on the order of $100 \mathrm{GHz}$ with state-of-the-art photodetectors [15,81-85], and the encoding of the input vector would be outside the WBAC system such that the latter would be completely passive during a given computation, not consuming any energy. The latter implies that the advantage in energy efficiency over an electronic computer would scale as $\mathcal{N}^{2}$. Ultimately, these promises have to be traded off against less-favorable noise levels, resulting effective truncation errors, and the difficulty of accessing the computation outputs' phase information.

\section{CONCLUSION}

We have demonstrated experimentally that any random medium can be employed as a reconfigurable wave-based analog computation unit, subject to appropriate wave-front shaping. Our proposal removes the hitherto crux of implementing wave-based analog computation in practice: the need for intricate metamaterial designs that are difficult (if not impossible so far) to fabricate. Moreover, our unit is reconfigurable to perform a different operation, without any additional calibration measurements. Our experimental demonstration leveraging infrastructure analogous to standard wireless indoor communication systems illustrates the applicability of our principle to all types of wave phenomena and random media, as well as the ease of practical implementation.

Our scheme's practicality and reconfigurability may play important roles in the renaissance of specific-purpose computation, heralded, in particular, by the rapidly expanding utilization of artificial neural networks, demanding high-speed low-power processing of big data. The reconfigurability of artificial neural networks is key to enable transfer learning as well as to correct errors of previous training phases [86]. Future versions of our concept can be extended to performing nonlinear operations [18] and thereby become themselves a complete, tailored hardware architecture for implementations of artificial neural networks [15,34], which may include cascading several reconfigurable cavities, possibly connected via nonlinear elements. There may also be possibilities to leverage the intrinsic IM interelement long-range correlations that are so far considered an obstacle. Furthermore, an exciting avenue for our concept is an implementation using optical multimode waveguides offering a stable and energy-efficient random medium for which wave-front shaping has become a well-mastered technique [87].

The raw data are available in Ref. [88].

\section{ACKNOWLEDGMENTS}

We thank Sébastien Popoff and Laurent Daudet for fruitful discussions and the anonymous referees for raising important questions. P. d. H. acknowledges funding from the French "Ministère de la Défense, Direction Générale de l'Armement".

The project was initiated, conceptualized, conducted, and written up by P. d. H. The metasurface was contributed by G. L. Both authors discussed the project.

[1] D. R. Solli and B. Jalali, Analog Optical Computing, Nat. Photonics 9, 704 (2015).

[2] G. E. Moore, Cramming More Components onto Integrated Circuits, Electronics 38, 114 (1965); Reprint available at https://ieeexplore.ieee.org/document/4785860.

[3] H. Esmaeilzadeh, E. Blem, R. S. Amant, K. Sankaralingam, and D. Burger, Dark Silicon and the End of Multicore Scaling, in Proceedings of the 38th Annual International Symposium on Computer Architecture (ISCA) (IEEE, New York, 2011), pp. 365-376, https://ieeexplore.ieee .org/abstract/document/6307773.

[4] S. K. Esser, P. A. Merolla, J. V. Arthur, A. S. Cassidy, R. Appuswamy, A. Andreopoulos, D. J. Berg, J. L. McKinstry, T. Melano, D. R. Barch, C. di Nolfo, P. Datta, A. Amir, B. Taba, M. D. Flickner, and D. S. Modha, Convolutional Networks for Fast, Energy-Efficient Neuromorphic Computing, Proc. Natl. Acad. Sci. U.S.A. 113, 11441 (2016).

[5] A. Graves et al., Hybrid Computing Using a Neural Network with Dynamic External Memory, Nature (London) 538, 471 (2016).

[6] J. Goodman, Introduction to Fourier Optics (McGraw-Hill, New York, 1996).

[7] M. Reck, A. Zeilinger, H. J. Bernstein, and P. Bertani, Experimental Realization of Any Discrete Unitary Operator, Phys. Rev. Lett. 73, 58 (1994).

[8] T. Zhu, Y. Zhou, Y. Lou, H. Ye, M. Qiu, Z. Ruan, and S. Fan, Plasmonic Computing of Spatial Differentiation, Nat. Commun. 8, 15391 (2017).

[9] A. Silva, F. Monticone, G. Castaldi, V. Galdi, A. Alù, and N. Engheta, Performing Mathematical Operations with Metamaterials, Science 343, 160 (2014).

[10] F. Monticone, N. M. Estakhri, and A. Alù, Full Control of Nanoscale Optical Transmission with a Composite Metascreen, Phys. Rev. Lett. 110, 203903 (2013).

[11] A. Sihvola, Enabling Optical Analog Computing with Metamaterials, Science 343, 144 (2014).

[12] D. A. B. Miller, Self-Configuring Universal Linear Optical Component, Photonics Res. 1, 1 (2013).

[13] J. Carolan et al., Universal Linear Optics, Science 349, 711 (2015).

[14] A. Ribeiro, A. Ruocco, L. Vanacker, and W. Bogaerts, Demonstration of a $4 \times 4$-Port Universal Linear Circuit, Optica 3, 1348 (2016).

[15] Y. Shen, N. C. Harris, S. Skirlo, M. Prabhu, T. Baehr-Jones, M. Hochberg, X. Sun, S. Zhao, H. Larochelle, D. Englund, 
and M. Soljačić, Deep Learning with Coherent Nanophotonic Circuits, Nat. Photonics 11, 441 (2017).

[16] D. A. B. Miller, Silicon Photonics: Meshing Optics with Applications, Nat. Photonics 11, 403 (2017).

[17] I. Goodfellow, Y. Bengio, and A. Courville, Deep Learning (MIT, Cambridge, MA, 2016).

[18] A. Li, S. Kim, Y. Luo, Y. Li, J. Long, and D. F. Sievenpiper, High-Power Transistor-Based Tunable and Switchable Metasurface Absorber, IEEE Trans. Microwave Theory Tech. 65, 2810 (2017).

[19] I. Freund, Looking through Walls and around Corners, Physica (Amsterdam) 168A, 49 (1990).

[20] M. Fink, Time Reversed Acoustics, Phys. Today 50, No. 3, 34 (1997).

[21] I. M. Vellekoop and A. P. Mosk, Focusing Coherent Light through Opaque Strongly Scattering Media, Opt. Lett. 32, 2309 (2007).

[22] A. P. Mosk, A. Lagendijk, G. Lerosey, and M. Fink, Controlling Waves in Space and Time for Imaging and Focusing in Complex Media, Nat. Photonics 6, 283 (2012).

[23] S. Rotter and S. Gigan, Light Fields in Complex Media: Mesoscopic Scattering Meets Wave Control, Rev. Mod. Phys. 89, 015005 (2017).

[24] S. M. Popoff, G. Lerosey, R. Carminati, M. Fink, A. C. Boccara, and S. Gigan, Measuring the Transmission Matrix in Optics: An Approach to the Study and Control of Light Propagation in Disordered Media, Phys. Rev. Lett. 104, 100601 (2010).

[25] J. Aulbach, B. Gjonaj, P. M. Johnson, A. P. Mosk, and A. Lagendijk, Control of Light Transmission through Opaque Scattering Media in Space and Time, Phys. Rev. Lett. 106, 103901 (2011).

[26] O. Katz, E. Small, Y. Bromberg, and Y. Silberberg, Focusing and Compression of Ultrashort Pulses through Scattering Media, Nat. Photonics 5, 372 (2011).

[27] P. del Hougne, F. Lemoult, M. Fink, and G. Lerosey, Spatiotemporal Wave Front Shaping in a Microwave Cavity, Phys. Rev. Lett. 117, 134302 (2016).

[28] I. M. Vellekoop, A. Lagendijk, and A. P. Mosk, Exploiting Disorder for Perfect Focusing, Nat. Photonics 4, 320 (2010).

[29] Y. Choi, T. D. Yang, C. Fang-Yen, P. Kang, K. J. Lee, R. R. Dasari, M. S. Feld, and W. Choi, Overcoming the Diffraction Limit Using Multiple Light Scattering in a Highly Disordered Medium, Phys. Rev. Lett. 107, 023902 (2011).

[30] J.-H. Park, C. Park, H. S. Yu, J. Park, S. Han, J. Shin, S. H. Ko, K. T. Nam, Y.-H. Cho, and Y. K. Park, Subwavelength Light Focusing Using Random Nanoparticles, Nat. Photonics 7, 454 (2013).

[31] S. R. Huisman, T. J. Huisman, T. A. W. Wolterink, A. P. Mosk, and P. W. H. Pinkse, Programmable Multiport Optical Circuits in Opaque Scattering Materials, Opt. Express 23, 3102 (2015).

[32] H. Defienne, M. Barbieri, I. A. Walmsley, B. J. Smith, and S. Gigan, Two-Photon Quantum Walk in a Multimode Fiber, Sci. Adv. 2, e1501054 (2016).

[33] R. Fickler, M. Ginoya, and R. W. Boyd, Custom-Tailored Spatial Mode Sorting by Controlled Random Scattering, Phys. Rev. B 95, 161108 (2017).
[34] A. Saade, F. Caltagirone, I. Carron, L. Daudet, A. Drémeau, S. Gigan, and F. Krzakala, Random Projections through Multiple Optical Scattering: Approximating Kernels at the Speed of Light, in Proceedings of the IEEE International Conference on Acoustics, Speech and Signal Processing (ICASSP) (IEEE, New York, 2016), pp. 6215-6219, https:// ieexplore.ieee.org/abstract/document/7472872.

[35] S. H. Simon, A. L. Moustakas, M. Stoytchev, and H. Safar, Communication in a Disordered World, Phys. Today 54, No. 9, 38 (2001).

[36] M. Kim, W. Choi, Y. Choi, C. Yoon, and W. Choi, Transmission Matrix of a Scattering Medium and Its Applications in Biophotonics, Opt. Express 23, 12648 (2015).

[37] H.-J. Stöckmann, Quantum Chaos: An Introduction (Cambridge University Press, Cambridge, England, 2007).

[38] J. N. Gollub et al., Large Metasurface Aperture for Millimeter Wave Computational Imaging at the Human-Scale, Sci. Rep. 7, 42650 (2017).

[39] T. Sleasman, M. F. Imani, J. N. Gollub, and D. R. Smith, Microwave Imaging Using a Disordered Cavity with a Dynamically Tunable Impedance Surface, Phys. Rev. Applied 6, 054019 (2016).

[40] M. Asefi and J. LoVetri, Use of Field-Perturbing Elements to Increase Nonredundant Data for Microwave Imaging Systems, IEEE Trans. Microwave Theory Tech. 65, 3172 (2017).

[41] P. del Hougne, M. F. Imani, T. Sleasman, J. N. Gollub, M. Fink, G. Lerosey, and D. R. Smith, Dynamic Metasurface Aperture as Smart Around-the-Corner Motion Detector, Sci. Rep. 8 (2018).

[42] P. del Hougne, M. F. Imani, M. Fink, D. R. Smith, and G. Lerosey, Precise Localization of Multiple Noncooperative Objects in a Disordered Cavity by Wave Front Shaping, Phys. Rev. Lett. 121, 063901 (2018).

[43] S. K. Hong, V. M. Mendez, T. Koch, W. S. Wall, and S. M. Anlage, Nonlinear Electromagnetic Time Reversal in an Open Semireverberant System, Phys. Rev. Applied 2, 044013 (2014).

[44] D. R. Smith, V. R. Gowda, O. Yurduseven, S. Larouche, G. Lipworth, Y. Urzhumov, and M. S. Reynolds, An Analysis of Beamed Wireless Power Transfer in the Fresnel Zone Using a Dynamic, Metasurface Aperture, J. Appl. Phys. 121, 014901 (2017).

[45] P. del Hougne, M. Fink, and G. Lerosey, Shaping Microwave Fields Using Nonlinear Unsolicited Feedback: Application to Enhance Energy Harvesting, Phys. Rev. Applied 8, 061001 (2017).

[46] V. R. Gowda, M. F. Imani, T. Sleasman, O. Yurduseven, and D. R. Smith, Focusing Microwaves in the Fresnel zone with a Cavity-Backed Holographic Metasurface, IEEE Access 6 , 12815 (2018).

[47] D. A. Hill, Electromagnetic Fields in Cavities: Deterministic and Statistical Theories (Wiley, New York, 2009), Vol. 35.

[48] J. Stein, H.-J. Stöckmann, and U. Stoffregen, Microwave Studies of Billiard Green Functions and Propagators, Phys. Rev. Lett. 75, 53 (1995).

[49] J. Barthélemy, O. Legrand, and F. Mortessagne, Complete S Matrix in a Microwave Cavity at Room Temperature, Phys. Rev. E 71, 016205 (2005). 
[50] S. Hemmady, X. Zheng, T. M. Antonsen, Jr., E. Ott, and S. M. Anlage, Universal Statistics of the Scattering Coefficient of Chaotic Microwave Cavities, Phys. Rev. E 71, 056215 (2005).

[51] U. Kuhl, H.-J. Stöckmann, and R. Weaver, Classical Wave Experiments on Chaotic Scattering, J. Phys. A 38, 10433 (2005).

[52] H. Weyl, Über die Asymptotische Verteilung der Eigenwerte, Nachr. Ges. Wiss. Goettingen Math.-Phys. Kl. 1911, 110 (1911).

[53] W. Arendt, R. Nittka, W. Peter, and F. Steiner, Weyl's Law: Spectral Properties of the Laplacian in Mathematics and Physics (Wiley-VCH, Weinheim, 2009).

[54] M. Dupré, P. del Hougne, M. Fink, F. Lemoult, and G. Lerosey, Wave-Field Shaping in Cavities: Waves Trapped in a Box with Controllable Boundaries, Phys. Rev. Lett. 115, 017701 (2015).

[55] N. Kaina, M. Dupré, G. Lerosey, and M. Fink, Shaping Complex Microwave Fields in Reverberating Media with Binary Tunable Metasurfaces, Sci. Rep. 4, 6693 (2014).

[56] P. del Hougne, M. Fink, and G. Lerosey, Optimal Communication Channels in a Disordered World with Tamed Randomness, arXiv:1810.00430.

[57] D. Sievenpiper, L. Zhang, R. F. J. Broas, N. G. Alexopolous, and E. Yablonovitch, High-Impedance Electromagnetic Surfaces with a Forbidden Frequency Band, IEEE Trans. Microwave Theory Tech. 47, 2059 (1999).

[58] D. F. Sievenpiper, J. H. Schaffner, H. J. Song, R. Y. Loo, and G. Tangonan, Two-Dimensional Beam Steering Using an Electrically Tunable Impedance Surface, IEEE Trans. Antennas Propag. 51, 2713 (2003).

[59] A. Sihvola, Metamaterials in Electromagnetics, Metamaterials 1, 2 (2007).

[60] N. Kaina, M. Dupré, M. Fink, and G. Lerosey, Hybridized Resonances to Design Tunable Binary Phase Metasurface Unit Cells, Opt. Express 22, 18881 (2014).

[61] A. Li, S. Singh, and D. Sievenpiper, Metasurfaces and Their Applications, Nanophotonics 7, 989 (2018).

[62] See Supplemental Material at http://link.aps.org/ supplemental/10.1103/PhysRevX.8.041037 for additional details on the experimental setup, the long-range correlations in the impact matrix, ensemble averaging over realizations, the time-sequential WBAC scheme, energy efficiency and notation.

[63] P. W. Anderson, Absence of Diffusion in Certain Random Lattices, Phys. Rev. 109, 1492 (1958).

[64] J. Wang and A. Z. Genack, Transport through Modes in Random Media, Nature (London) 471, 345 (2011).

[65] A. Derode, A. Tourin, and M. Fink, Random Multiple Scattering of Ultrasound. II. Is Time Reversal a SelfAveraging Process?, Phys. Rev. E 64, 036606 (2001).

[66] N. D. Sidiropoulos, T. N. Davidson, and Z.-Q. Luo, Transmit Beamforming for Physical-Layer Multicasting, IEEE Trans. Signal Process. 54, 2239 (2006).

[67] S. Sen, B. Radunovic, R. R. Choudhury, and T. Minka, You are Facing the Mona Lisa: Spot Localization Using PHY Layer Information, in Proceedings of the 10th International Conference on Mobile Systems, Applications, and Services (Association for Computing Machinery, New York, 2012), pp. 183-196, https://dl.acm.org/citation.cfm?doid=2307636 .2307654 .

[68] H. Yang, X. Cao, F. Yang, J. Gao, S. Xu, M. Li, X. Chen, Y. Zhao, Y. Zheng, and S. Li, A Programmable Metasurface with Dynamic Polarization, Scattering and Focusing Control, Sci. Rep. 6, 35692 (2016).

[69] P. del Hougne, B. Rajaei, L. Daudet, and G. Lerosey, Intensity-Only Measurement of Partially Uncontrollable Transmission Matrix: Demonstration with Wave-Field Shaping in a Microwave Cavity, Opt. Express 24, 18631 (2016).

[70] Interestingly, a similar scaling law proportional to $\mathcal{N}^{2}$ is found for the number of components of a photonic circuit consisting of beam splitters to implement a desired $\mathcal{N} \times \mathcal{N}$ linear operation $[7,15]$.

[71] A. Drémeau, A. Liutkus, D. Martina, O. Katz, C. Schülke, F. Krzakala, S. Gigan, and L. Daudet, Reference-less Measurement of the Transmission Matrix of a Highly Scattering Material Using a DMD and Phase Retrieval Techniques, Opt. Express 23, 11898 (2015).

[72] I. M. Vellekoop and A. P. Mosk, Phase Control Algorithms for Focusing Light through Turbid Media, Opt. Commun. 281, 3071 (2008).

[73] D. Bertsimas and O. Nohadani, Robust Optimization with Simulated Annealing, J. Global Optim. 48, 323 (2010).

[74] J. Peurifoy, Y. Shen, L. Jing, Y. Yang, F. Cano-Renteria, B. G. DeLacy, J. D. Joannopoulos, M. Tegmark, and M. Soljačić, Nanophotonic Particle Simulation and Inverse Design Using Artificial Neural Networks, Sci. Adv. 4, eaar4206 (2018).

[75] E. G. van Putten, D. Akbulut, J. Bertolotti, W. L. Vos, A. Lagendijk, and A. P. Mosk, Scattering Lens Resolves Sub-100 nm Structures with Visible Light, Phys. Rev. Lett. 106, 193905 (2011).

[76] V. Sze, Y.-H. Chen, T.-J. Yang, and J. S. Emer, Efficient Processing of Deep Neural Networks: A Tutorial and Survey, Proc. IEEE 105, 2295 (2017).

[77] Y. Jia, E. Shelhamer, J. Donahue, S. Karayev, J. Long, R. Girshick, S. Guadarrama, and T. Darrell, Caffe: Convolutional Architecture for Fast Feature Embedding, in Proceedings of the 22nd ACM International Conference on Multimedia (Association for Computing Machinery, New York, 2014), pp. 675-678, https://dl.acm.org/citation .cfm?id=2654889.

[78] J.-B. Gros, P. del Hougne, and G. Lerosey, Les Cavités Micro-Ondes Reconfigurables: Une Nouvelle Approche pour Fabriquer des Chambres Réverbérantes Chaotiques, in Proceedings of the 19ème Colloque International \& Exposition sur la Compatibilité ElectroMagnétique (unpublished).

[79] R. Serra, A. C. Marvin, F. Moglie, V. M. Primiani, A. Cozza, L. R. Arnaut, Y. Huang, M. O. Hatfield, M. Klingler, and F. Leferink, Reverberation Chambers à la Carte: An Overview of the Different Mode-Stirring Techniques, IEEE Electromagn. Compat. Mag. 6, 63 (2016).

[80] M. Horowitz, 1.1 Computing's Energy Problem (and What We Can Do about It), in 2014 IEEE International SolidState Circuits Conference Digest of Technical Papers (ISSCC) (IEEE, New York, 2014), pp. 10-14. 
[81] L. Vivien, A. Polzer, D. Marris-Morini, J. Osmond, J. M. Hartmann, P. Crozat, E. Cassan, C. Kopp, H. Zimmermann, and J. M. Fédéli, Zero-Bias 40 Gbit/s Germanium Waveguide Photodetector on Silicon, Opt. Express 20, 1096 (2012).

[82] Y. Salamin, P. Ma, B. Baeuerle, A. Emboras, Y. Fedoryshyn, W. Heni, B. Cheng, A. Josten, and J. Leuthold, $100 \mathrm{GHz}$ Plasmonic Photodetector, ACS Photonics 5, 3291 (2018).

[83] Y. Ding, Z. Cheng, X. Zhu, K. Yvind, J. Dong, M. Galili, H. Hu, N. A. Mortensen, S. Xiao, and L. K. Oxenløwe, Ultra-compact Graphene Plasmonic Photodetector with the Bandwidth over $110 \mathrm{GHz}$, arXiv:1808.04815.

[84] Finisar $100 \mathrm{GHz}$ single high-speed photodetector, https:// www.finisar.com/communication-components/xpdv412xr.
[85] C. Wang, M. Zhang, X. Chen, M. Bertrand, A. Shams-Ansari, S. Chandrasekhar, P. Winzer, and M. Lončar, Integrated Lithium Niobate Electro-Optic Modulators Operating at CMOS-Compatible Voltages, Nature (London) 562, 101 (2018).

[86] L. Pratt and B. Jennings, A Survey of Connectionist Network Reuse through Transfer, in Learning to Learn (Springer, New York, 1996), pp. 19-43.

[87] P. Ambichl, W. Xiong, Y. Bromberg, B. Redding, H. Cao, and S. Rotter, Super-and Anti-Principal-Modes in Multimode Waveguides, Phys. Rev. X 7, 041053 (2017).

[88] P. del Hougne and G. Lerosey, Zenodo, DOI: 10.5281/ zenodo.1323344 (2018). 